\title{
Radio spectrum and distance of the SNR HB9
}

\author{
D. A. Leahy ${ }^{1}$ and W. W. Tian ${ }^{1,2}$ \\ 1 Department of Physics \& Astronomy, University of Calgary, Calgary, Alberta T2N 1N4, Canada \\ e-mail: wtian@ucalgary.ca \\ 2 National Astronomical Observatories, CAS, Beijing 100012, PR China \\ Received 23 June 2006 / Accepted 13 September 2006
}

ABSTRACT

\begin{abstract}
New images are presented of the supernova remnant (SNR) HB9 based on $408 \mathrm{MHz}$ and $1420 \mathrm{MHz}$ continuum emission and HI-line emission data from the Canadian Galactic Plane Survey (CGPS). Two different T-T plot methods and new integrated flux densities give spectral index $\left(S_{v} \propto v^{-\alpha}\right)$ for the whole of HB9 of $0.48 \pm 0.03 ; 0.49$; and $0.47 \pm 0.06$, respectively. These values are lower than the previous spectral index estimated for HB9 $(\alpha=0.61)$. The change is mainly due to improved $1420 \mathrm{MHz}$ data. No difference in spectral index is detected between strong and weak filaments. A new result is that the spectral index for interior regions is steeper than for the rim. This can be explained by a standard curved interstellar electron energy spectrum combined with lower interior magnetic field compared to that near the outer shock. This results in a larger proportion of steep spectrum emission for lines-ofsight through the central body of the SNR. HI observations show structures probably associated with the SNR in the radial velocity range -3 to $-9 \mathrm{~km} \mathrm{~s}^{-1}$, suggesting a kinematic distance of $0.8 \pm 0.4 \mathrm{kpc}$ for the SNR. This is consistent with the distance to the radio pulsar PSR B0458+46, offset from the center of HB9 by $23^{\prime}$. However the pulsar spindown and kinematic ages are significantly greater than estimates of the SNR age: the Sedov age for HB9 is $6600 \mathrm{yr}$ and the evaporative cloud model yields ages of 4000-7000 yr.
\end{abstract}

Key words. radio continuum: ISM - radio lines: ISM - ISM: supernova remnants

\section{Introduction}

The production of high-energy particles in our Galaxy is closely related to shock acceleration in supernova remnants (SNR). The study of the radio spectra produced by high-energy electrons in SNRs allows one to learn about the electron energy spectrum. Due to its large diameter HB9 is one of the best candidates for a study of SNRs' spectral index spatial variations. Variations have been observed to occur in some other large angular sized SNRs (Leahy \& Tian 2005; Tian \& Leahy 2005; Uyaniker et al. 2004; Alvarez et al. 2001). Spatial variations in the spectral index across HB9 has been previously noted by Leahy et al. (1998) and Leahy \& Roger (1991), but a new set of higher resolution and sensitivity observations of the Canadian Galactic Plane Survey (CGPS) combined with new spectral index analysis methods, impel us to carry out a new spectral index study of HB9. A lack of direct estimates of the distance to HB9 in previous research (Leahy \& Roger 1991; Lozinskaya 1981; Milne 1979) is corrected here by an analysis of the new HI observations of HB9.

\section{Observations and image analysis}

The continuum and HI emission data sets come from the CGPS, described in detail by Taylor et al. (2003). The data sets are mainly based on observations from the Synthesis Telescope (ST) of the Dominion Radio Astrophysical Observatory (DRAO). The spatial resolution of the continuum images of HB9 is $49^{\prime \prime} \times 68^{\prime \prime}$ at $1420 \mathrm{MHz}$ and $2.8^{\prime} \times 3.9^{\prime}$ at $408 \mathrm{MHz}$. The synthesized beam for the HI line images is $58^{\prime \prime} \times 80^{\prime \prime}$ and the radial velocity resolution is $1.32 \mathrm{~km} \mathrm{~s}^{-1}$. The continuum images are noise limited with $\mathrm{rms}$ of $\sim 0.3 \mathrm{mJy} /$ beam at $1420 \mathrm{MHz}$ and $\sim 3 \mathrm{mJy} /$ beam at $408 \mathrm{MHz}$. The DRAO ST observations are not sensitive to structures larger than an angular size scale of about $3.3^{\circ}$ at $408 \mathrm{MHz}$ and 56' at $1420 \mathrm{MHz}$. Thus the CGPS includes data from the $408 \mathrm{MHz}$ all-sky survey of Haslam et al. (1982), sensitive to structure greater than $51^{\prime}$, and the Effelsberg $1.4 \mathrm{GHz}$ Galactic plane survey of Reich et al. (1990, 1997), sensitive to structure greater than $9.4^{\prime}$. The large scale HI data is from the single-antenna survey of the CGPS area (Higgs \& Tapping 2000) with resolution of $36^{\prime}$.

There are many compact sources (CS) overlapping the face of HB9. In order to better study the spectral index distribution, we compare two methods to reduce the effects of CS on the SNR's spectral index. The first removes CS at both frequencies but is limited to the resolution of the $408 \mathrm{MHz}$ image; the other removes CS only in the $1420 \mathrm{MHz}$ image so is effective at higher spatial resolution. The first method was introduced by Tian \& Leahy (2005) and the second was introduced by Leahy (2006). Both have been proved effective in removing CS contamination. We also analyze HI spectral line observations of HB9 and estimate HB9's distance.

\section{Results}

\subsection{HB9 flux densities at $408 \mathrm{MHz}$ and $1420 \mathrm{MHz}$}

The first row of Fig. 1 shows the $408 \mathrm{MHz}$ (left) and $1420 \mathrm{MHz}$ (right) images of HB9 overlaid by boxes used for spectral index determination. The $408 \mathrm{MHz}$ and $1420 \mathrm{MHz}$ CS-subtracted images are shown in the second row of Fig. 1. $160 \mathrm{CS}$ at $1420 \mathrm{MHz}$ and $61 \mathrm{CS}$ at $408 \mathrm{MHz}$ are detected within HB9. The total integrated flux densities of these CS are $12.0 \mathrm{Jy}$ at $408 \mathrm{MHz}$ and $5.6 \mathrm{Jy}$ at $1420 \mathrm{MHz}$. After subtracting the flux of CS, HB9's integrated flux density is $117.8 \pm 5.3 \mathrm{Jy}$ at $408 \mathrm{MHz}$ and $65.9 \pm 3.4 \mathrm{Jy}$ at $1420 \mathrm{MHz}$. This gives an integrated flux-density 

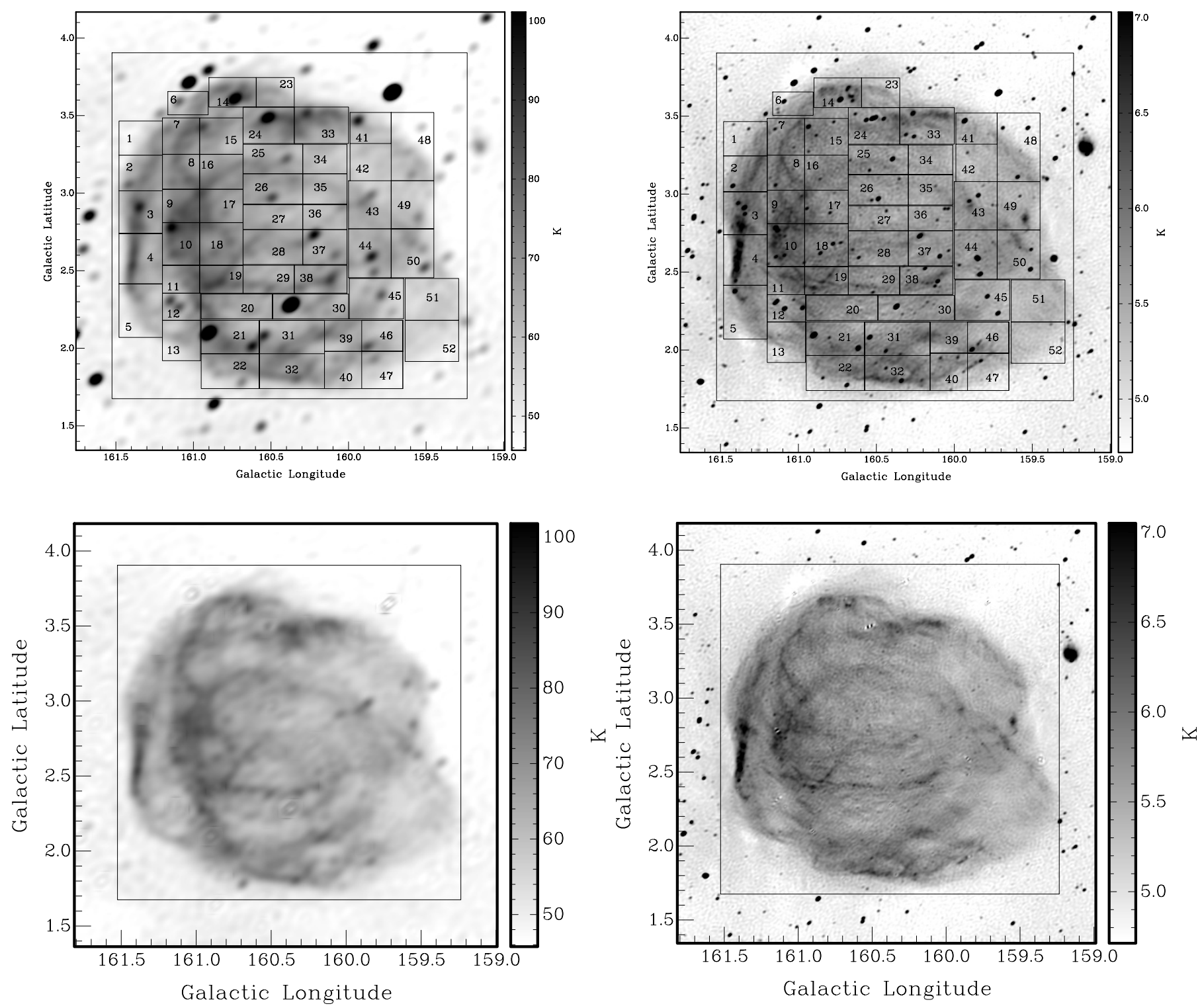

Fig. 1. The first row shows HB9 at $408 \mathrm{MHz}$ (left) and $1420 \mathrm{MHz}$ (right). The second row shows the $408 \mathrm{MHz}$ image with compact sources (CS) subtracted (left) and the $1420 \mathrm{MHz}$ image with CS subtracted (right). The single large box is the area used for T-T plots of the whole of HB9; the 52 small boxes labeled with numbers show areas used for T-T plots of subareas of HB9.

408-1420 MHz spectral index $\alpha=0.48 \pm 0.06$ including $\mathrm{CS}$, and $0.47 \pm 0.06$ after subtracting $\mathrm{CS}$. The results are consistent within errors.

\subsection{HB9 T-T plot spectral indices}

We have applied the T-T plot method to investigate spectral indices (e.g. Tian \& Leahy 2005). The T-T plot method is first applied to the whole area covering HB9 (the single big box of Fig. 1). Figure 2 gives the $408-1420 \mathrm{MHz}$ T-T plots for three cases (from left to right): case 1 - maps including CS ( $\alpha_{\text {auto }}=$ $0.50 \pm 0.03$ ); case $2-\mathrm{CS}$ subtracted from both $408 \mathrm{MHz}$ and $1420 \mathrm{MHz}$ maps $\left(\alpha_{\text {auto }}=0.48 \pm 0.03\right)$; case 3- maps with Gaussian fits to CS subtracted from the $1420 \mathrm{MHz}$ map only ( $\left.\alpha_{\text {manual }}=0.49\right)$. The detailed description of case 3 is given in Leahy (2006): the motivation is two-fold: to remove CS which are only clearly resolved at $1420 \mathrm{MHz}$ and not at $408 \mathrm{MHz}$; and to be able to clearly separate points in the T-T plot which belong to CS from points which belong to diffuse SNR emission. The subscript auto refers to the case of an automated linear fit including all of the points, the subscript manual refers to the case of a manual fit done to the points excluding points due to CS. The points due to CS are clearly seen in the left plot of Fig. 2 if they have a significantly different slope than the points from the SNR. For the middle plot, CS are subtracted at both 408 and $1420 \mathrm{MHz}$. However, there are artifacts due to some CS subtracted at $1420 \mathrm{MHz}$ but not at $408 \mathrm{MHz}$ and vice versa. For the right plot, since CS are subtracted only at $1420 \mathrm{MHz}$, points due to CS show up as vertical lines of points, with $408 \mathrm{MHz}$ flux but no $1420 \mathrm{MHz}$ flux. This occurs even if a CS has a spectral index similar to the diffuse SNR emission.

To study spatial variations in HB9, we divide it into 52 regions (see the top plots of Fig. 1). The case 3 method removes fainter CS detected only at $1420 \mathrm{MHz}$ (for discussion, see Leahy 2006). We give the results in Table 1 and for comparison, the spectral index from case 1 including CS is also given. Table 1 shows that case 1 and case 3 results can be quite different. Figure 3 shows T-T plots for region 21: for case 1 (left, 

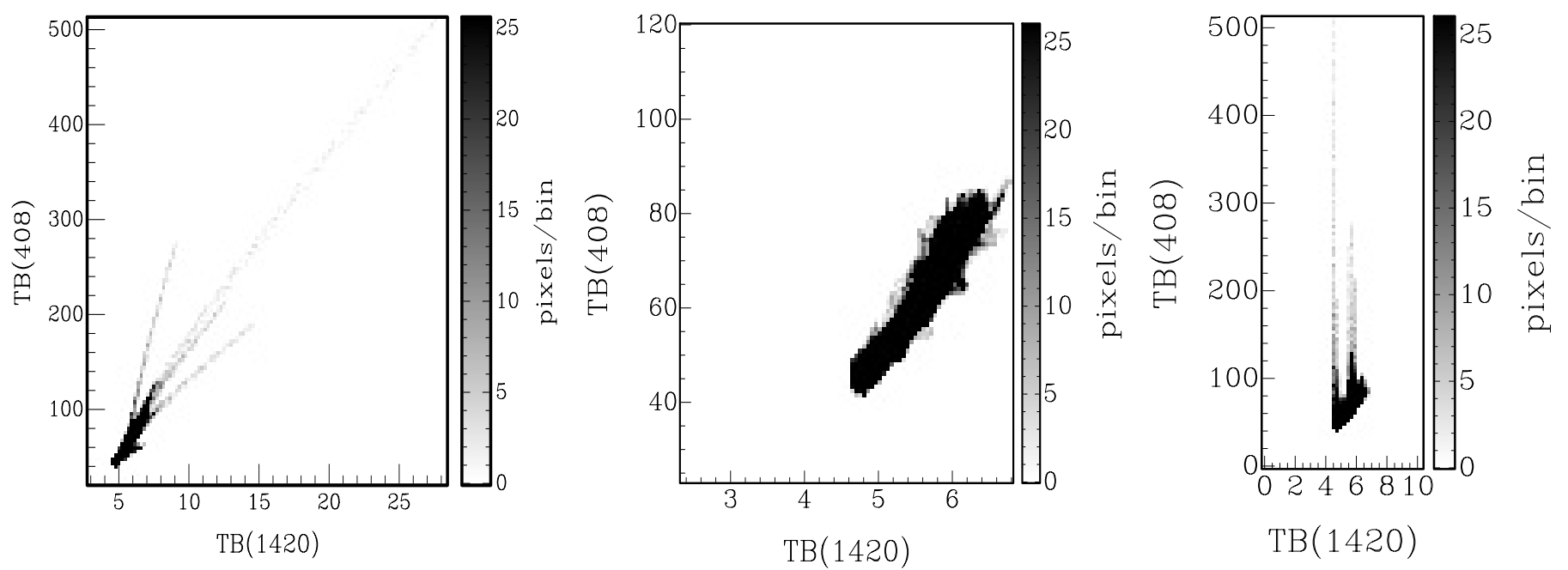

Fig. 2. 408-1420 MHz T-T plots for the whole of HB9: including CS (left, $\alpha_{\text {auto }}=0.50 \pm 0.03$ ); for CS subtracted from both $408 \mathrm{MHz}$ and $1420 \mathrm{MHz}$ maps (middle, $\alpha_{\text {auto }}=0.48 \pm 0.03$ ); for CS subtracted from $1420 \mathrm{MHz}$ map only $\left(\right.$ right, $\alpha_{\text {manual }}=0.49$ ).

Table 1. HB9 408-1420 MHz spectral index for regions 1 to 52.

\begin{tabular}{cccccc}
\hline \hline Region & $\alpha_{\text {case } 1}$ & $\alpha_{\text {case3 }}$ & Region & $\alpha_{\text {case } 1}$ & $\alpha_{\text {case } 3}$ \\
\hline 1 & $0.58 \pm 0.01$ & 0.55 & 27 & $0.75 \pm 0.07$ & 0.65 \\
2 & $0.51 \pm 0.01$ & 0.52 & 28 & $0.85 \pm 0.11$ & 0.76 \\
3 & $0.55 \pm 0.02$ & 0.53 & 29 & $1.05 \pm 0.08$ & 0.97 \\
4 & $0.50 \pm 0.01$ & 0.47 & 30 & $1.38 \pm 0.01$ & 0.51 \\
5 & $0.44 \pm 0.04$ & 0.50 & 31 & $0.75 \pm 0.02$ & 0.53 \\
6 & $0.65 \pm 0.09$ & 0.53 & 32 & $0.52 \pm 0.02$ & 0.40 \\
7 & $0.85 \pm 0.09$ & 0.63 & 33 & $0.82 \pm 0.03$ & 0.74 \\
8 & $0.87 \pm 0.09$ & 0.55 & 34 & $0.87 \pm 0.11$ & 0.65 \\
9 & $0.86 \pm 0.03$ & 0.76 & 35 & $0.81 \pm 0.11$ & 0.69 \\
10 & $0.72 \pm 0.10$ & 0.60 & 36 & $0.77 \pm 0.02$ & 0.72 \\
11 & $0.78 \pm 0.05$ & 0.52 & 37 & $0.77 \pm 0.03$ & 0.63 \\
12 & $0.52 \pm 0.05$ & 0.54 & 38 & $0.81 \pm 0.02$ & 0.80 \\
13 & $0.57 \pm 0.04$ & 0.46 & 39 & $0.83 \pm 0.04$ & 0.69 \\
14 & $0.69 \pm 0.02$ & 0.70 & 40 & $0.29 \pm 0.05$ & 0.33 \\
15 & $0.91 \pm 0.03$ & 0.96 & 41 & $0.42 \pm 0.02$ & 0.41 \\
16 & $0.93 \pm 0.03$ & 0.91 & 42 & $0.55 \pm 0.23$ & 0.55 \\
17 & $0.75 \pm 0.02$ & 0.56 & 43 & $0.77 \pm 0.03$ & 0.73 \\
18 & $0.77 \pm 0.10$ & 0.63 & 44 & $0.56 \pm 0.04$ & 0.61 \\
19 & $0.88 \pm 0.07$ & 0.79 & 45 & $0.52 \pm 0.02$ & 0.55 \\
20 & $0.85 \pm 0.07$ & 0.77 & 46 & $0.53 \pm 0.02$ & 0.55 \\
21 & $0.51 \pm 0.01$ & 0.61 & 47 & $0.35 \pm 0.02$ & 0.29 \\
22 & $0.50 \pm 0.02$ & 0.48 & 48 & $0.51 \pm 0.02$ & 0.50 \\
23 & $0.72 \pm 0.02$ & 0.70 & 49 & $0.57 \pm 0.03$ & 0.46 \\
24 & $0.84 \pm 0.02$ & 0.73 & 50 & $0.76 \pm 0.03$ & 0.69 \\
25 & $0.70 \pm 0.09$ & 0.76 & 51 & $0.45 \pm 0.03$ & 0.40 \\
26 & $0.69 \pm 0.05$ & 0.66 & 52 & $0.70 \pm 0.14$ & 0.50 \\
\hline & & & & &
\end{tabular}

$\left.\alpha_{\text {auto }}=0.51 \pm 0.01\right)$, case 2 (middle, $\alpha_{\text {auto }}=0.88 \pm 0.11$ ), and case 3 (right, $\alpha_{\text {manual }}=0.61$ ). The spectral index for case 1 is dominated by the single bright CS. Case 2 gives an erroneous (too large) value of spectral index. The reason is that in the presence of diffuse emission, the Gaussian fitting to the strongest CS produces very low flux at $408 \mathrm{MHz}$, resulting in a residual flux in the CS subtracted map at this frequency. The advantage of case 3 is more apparent here, since there is no overcrowding of the CS in the T-T plot, like there was in Fig. 2 (right). This allows easy identification of the $\mathrm{CS}$, including the faint $\mathrm{CS}$ at $\mathrm{TB}(1420)=5.8 \mathrm{~K}$

A histogram of the T-T plot spectral index values from Table 1 is shown in Fig. 4. The dashed line shows the spectral indices obtained from automatic fits, including CS at both
$408 \mathrm{MHz}$ and $1420 \mathrm{MHz}$ (case 1); the solid line shows the spectral indices obtained from manual fits for CS subtracted at $1420 \mathrm{MHz}$ (case 3). The effect of CS on the spectral index distribution is clear: there is a second peak in the histogram introduced at 0.7 to 0.9 due to CS, whereas the SNR only emission (case 3) has a smooth distribution of indices between 0.4 and 0.8 .

\section{3. $\mathrm{HI}$ emission}

The CGPS data has 2 times better velocity resolution and better sensitivity than the older DRAO HI data (Leahy \& Roger 1991). We find HI emission which is spatially associated with the boundary of HB9 and a deficit of emission associated with the interior in the velocity range -3 to $-9 \mathrm{~km} \mathrm{~s}^{-1}$, but not at other velocities. This suggests that this HI is likely to be physically associated with the SNR. Figure 5 shows the average of the maps of HI emission in the 8 channels from -3 to $-9 \mathrm{~km} \mathrm{~s}^{-1}$, with a contour of continuum emission at $1420 \mathrm{MHz}$ to show the boundary of HB9.

\section{Discussion}

\subsection{Distance of HB9, age, and possible association with pulsar $0458+46$}

For the velocity range of -3 to $-9 \mathrm{~km} \mathrm{~s}^{-1}$, using circular galactic rotation velocity $V_{R}=220 \mathrm{~km} \mathrm{~s}^{-1}$ and solar distance $R_{0}=8.5 \mathrm{kpc}$ from the Galactic center, yields a distance to HB9 of $d=0.8 \pm$ $0.4 \mathrm{kpc}$. This distance is consistent within errors with previous estimates: greater than $1 \mathrm{kpc}$ derived from measurements of radial velocity of $\mathrm{H} \alpha$ filaments by Lozinskaya (1981); about $1.1 \mathrm{kpc}$ from the X-ray properties of HB9 by Leahy (1987); and $1.3-1.8 \mathrm{kpc}$ from surface-brightness-diameter relations by Milne (1979) and Caswell \& Lerche (1979).

Since the angular size of HB9 is $130^{\prime}$ by $120^{\prime}$, the mean radius is $15 \mathrm{pc}$ at $0.8 \mathrm{kpc}$. A Sedov model (Cox 1972) is applied, using the X-ray temperature and X-ray flux from Leahy \& Aschenbach (1995). For a $15 \mathrm{pc}$ radius, an age of $6600 \mathrm{yr}$ and a ratio between explosion energy $E_{51}$ (in $10^{51} \mathrm{erg}$ ) and initial density $n_{0}\left(\right.$ in $\left.^{-3}\right) E_{51} / n_{0}=5$ are obtained.

As noted before by Leahy \& Aschenbach (1995), based on the X-ray morphology and uniform temperature, it is likely that 

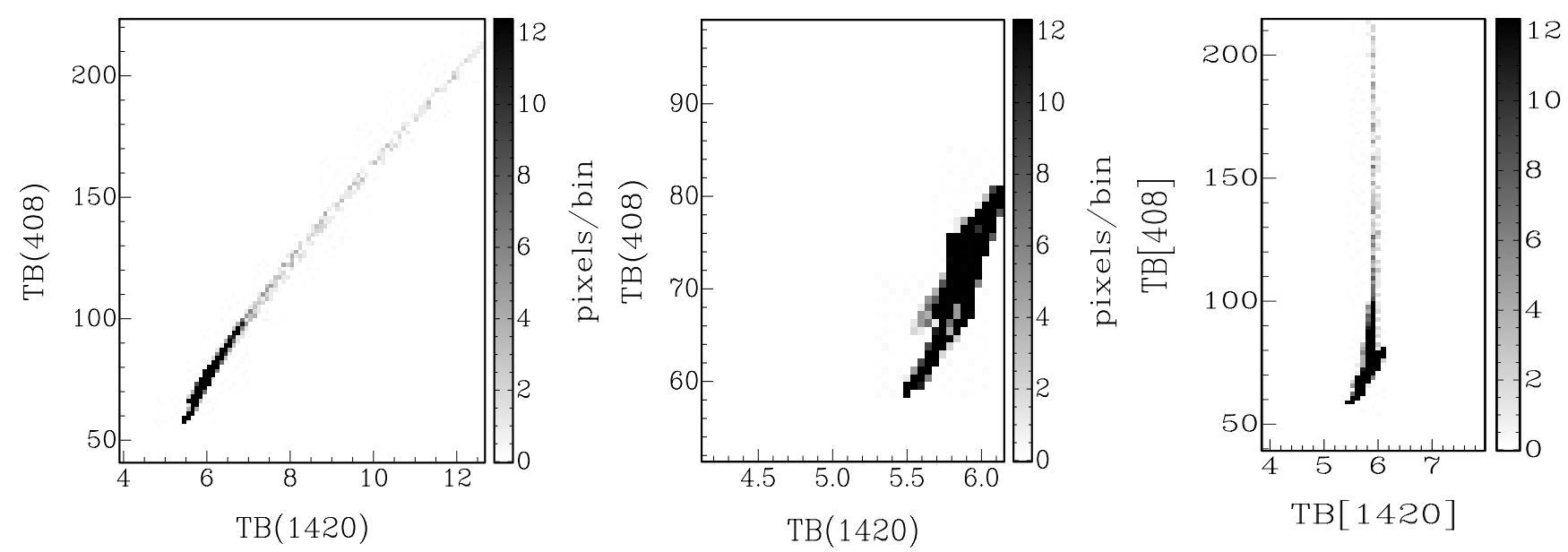

Fig. 3. 408-1420 MHz T-T plots of region 21: including CS (left); for CS subtracted from both $408 \mathrm{MHz}$ and $1420 \mathrm{MHz}$ maps (middle); for CS subtracted from $1420 \mathrm{MHz}$ map only (right).

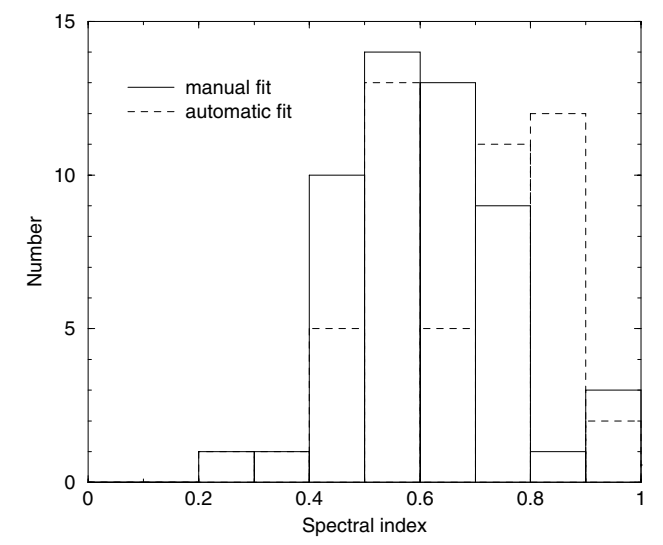

Fig. 4. Histogram of spectral indices in HB9: automatic fit includes compact sources (CS); manual fit excludes CS.

Sedov model does not apply in this case. The evaporative cooling model developed by White \& Long (1991) provides a better explanation for the observed X-ray features. If White \& Long's (1991) model is applied for a distance of $0.8 \mathrm{kpc}$, a range of parameters is found which give a nearly flat temperature profile at $0.8 \mathrm{keV}$ and an appropriately centrally brightened X-ray profile ( $\tau=10$ and $C=20$ to $C=50$ ). Then the preshock intercloud density is $3-10 \times 10^{-3} \mathrm{~cm}^{-3}$, the age is $4000-7000 \mathrm{yr}$ and the explosion energy is $0.15-0.3 \times 10^{51} \mathrm{erg}$.

The radio pulsar $0458+46$ is only $23^{\prime}$ from the center of HB9. It has a DM distance $1.8 \mathrm{kpc}$, spin-down age $1.8 \times 10^{6} \mathrm{yr}$ and transverse velocity $95.5 \mathrm{~km} \mathrm{~s}^{-1}$ (Manchester et al. 2005). The DM distance is expected to be larger than the distance derived from the HI data, due to the extra DM introduced by the extra electron density in the SNR shell. The spindown age is based on the assumption of a fast initial spin, so it probably overestimates the true age. The pulsar's kinematic age based on its transverse velocity is $5.5 \times 10^{4} \mathrm{yr}$, several times larger than HB9's age from either Sedov or evaporative cloud models. Thus an association between the pulsar and HB9 is possible, but only if it was a somewhat off-center explosion: for instance, the kinematic age can be reduced to about $7000 \mathrm{yr}$ if the explosion center is $\sim 3^{\prime}$ from the current pulsar position. Better X-ray observations of HB9 should determine the nature of SNR, and give an improved SNR age value.

\subsection{HB9 spectral index}

Based on T-T plots, Leahy \& Roger (1991) obtained a mean T-T plot spectral index of 0.61 for HB9. The new T-T plot spectral index derived for the whole SNR HB9 $(\alpha=0.48 \pm 0.03)$ is much smaller, but consistent with the integrated flux densitybased spectral index $(\alpha=0.47 \pm 0.06)$. Two main factors appear as responsible for this. First, there is a significant change (3-4 K difference) in SNR brightness temperature at $1420 \mathrm{MHz}$ compared to earlier data set. Figure 2 of Leahy \& Roger (1991) shows a bright filament at $\sim 3.2 \mathrm{~K}$ compared to $\sim 6.5 \mathrm{~K}$ in the current $1420 \mathrm{MHz}$ image, and no such change in the $408 \mathrm{MHz}$ images. Second, the present observations have higher resolution and sensitivity, so more compact sources have been resolved and subtracted from the images of HB9.

In what follows, we discuss the spectral index variations with location within HB9. Since the integrated flux and T-T plot spectral indices for the whole of HB9 agree each other, the filamentary emission (measured by the T-T plot method) and the total emission (filamentary plus spatially smooth emission) should have the same spectral index. To analyze local variations we divided the 52 regions into 3 groups: (a) boxes that cover regions with strong filamentary emission (those labeled with numbers 2 , $3,4,5,7,8,9,10,11,14,19,32,33,47,50$ ); (b) boxes overlapping weak filamentary emission (boxes 15, 16, 20, 26, 27, 30, $34,35,36,37,41,42,45,51,52)$; and (c) those with intermediate filamentary emission (the remainder). The means (and standard deviations) from the 3 groups were $0.58(0.14), 0.64(0.17)$ and $0.61(0.14)$, respectively. Thus there is no clear difference in spectral index between regions including strong filaments and regions with weak filaments. We can therefore conclude that the spectral results confirm the scenario suggested by Leahy $\&$ Roger (1991), where the filaments are edge-on viewing of emitting sheets.

Larger differences in spectrum are apparent from the comparison of the SNR periphery with regions in the interior. When one looks at spectral index ( $\alpha_{\text {case3 }}$ in Table 1$)$ vs. position, one finds that the flatter spectral indices $(<0.5)$ are all associated with the SNR limb and all of the steeper values $(>0.7)$ are associated with the interior. The mean and standard deviation of spectral indices for all limb regions are 0.49 and 0.11 , whereas the values for the interior regions are 0.67 and 0.12 , respectively. An alternate analysis of spectral index was carried out to verify this result. We made a spectral index map of HB9 using the 


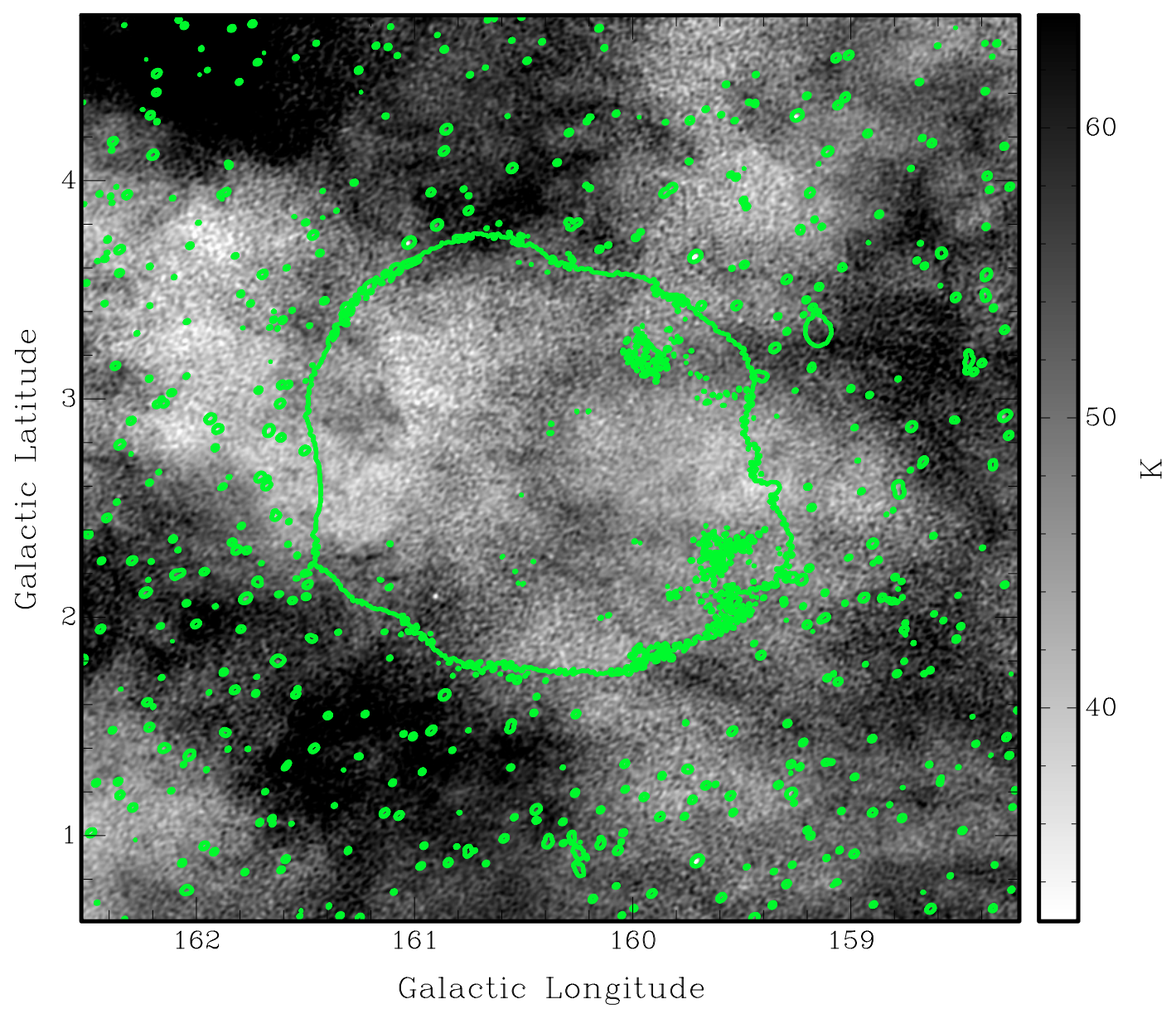

Fig. 5. $\mathrm{HI}$ emission in the field centered on HB9: average of velocity channels -3 to $-9 \mathrm{~km} \mathrm{~s}^{-1}$, with $1420 \mathrm{MHz}$ continuum contour at $5.3 \mathrm{~K} T_{B}$ indicating HB9. HI image is noise limited with rms brightness temperature $\delta T_{B} \sim 1 \mathrm{~K}$. The synthesized beam for the HI line images is $58^{\prime \prime} \times 80^{\prime \prime}$.
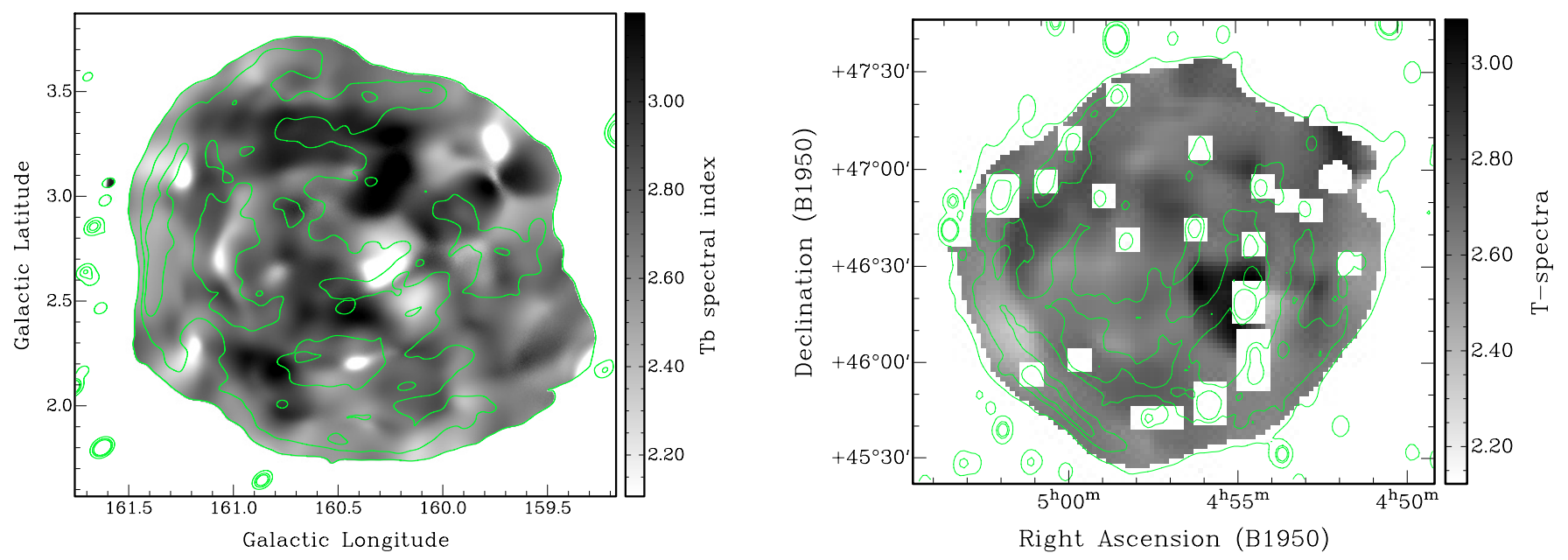

Fig. 6. 408-1420 MHz spectral index maps of HB9: from the current images with CS subtracted at both frequencies (left panel) with $1420 \mathrm{MHz}$ contours at 5.25, 5.7, 6.1 K; the spectral index map from the old 408 and $1420 \mathrm{MHz}$ images (right panel), same as Fig. 6 of Leahy et al. (1998).

running T-T plot method of Zhang et al. (1997): the method yields $T_{B}$ spectral index $\beta$, with $\beta=\alpha+2$. We used the CS subtracted images at $408 \mathrm{MHz}$ and $1420 \mathrm{MHz}$ to create the spectral index map shown in Fig. 6, left panel. The small white patches show the subregions where the fitting algorithm did not obtain a reliable value of spectral index. The spectral index map computed from images including CS is similar except it has a number of elliptical spots (one for each bright CS) at the locations of the $\mathrm{CS}$ and with shading corresponding to the CS spectral index. One can see from Fig. 6 (left) that the limb of HB9 has predominantly low spectral index and the interior has predominantly high spectral index. From the discussion here and in previous papers (e.g. Tian \& Leahy 2005; Leahy 2006), the reliability of the automatic fits used by the spectral map software is often poor, yet the map can give a general indication of spatial variations of spectral index. 
A general discussion of mechanisms that govern the radio spectrum is given in Leahy \& Roger (1998). Absorption processes and electron energy losses from ionization act more effectively at lower frequencies and result in spectral flattening. Synchrotron and inverse-Compton act at high energies and steepen the spectrum. If the electron energy spectrum is curved then spatial variations in spectral index can occur due to variations in magnetic field, which determines the observed synchrotron frequency for a given electron energy. The diffuse galactic electron spectrum has an electron energy index steepening from $\sim 2.0$ below $\sim 2 \mathrm{GeV}$ to $\sim 2.6$ above $\sim 5 \mathrm{GeV}$, then to $\sim 3.5$ above $\sim 40 \mathrm{GeV}$, corresponding to synchrotron index increasing from $\alpha=0.5$ at low frequencies to $\alpha=1.25$ at high frequencies. For a fixed observing frequency, increasing magnetic field, B, means sampling lower electron energies, so that spectral index flattens with increasing B.

For HB9, the steeper index for interior regions, for which the line of sight goes through the whole SNR, could be interpreted as due to lower B for the interior compared to the rim; stronger synchrotron losses for the interior; or stronger absorption effects at the rim. For the Cygnus Loop (Leahy \& Roger 1998) low frequency observations confirm that the NE rim shows the effects of thermal plasma absorption. However for HB9, previous studies have not found significant weakening of the radio brightness at the rim at low frequencies. This leaves the first two causes to examine more closely. The radio morphology of HB9 is consistent with a low brightness interior surrounded by a brighter shell with filaments: the image at $1420 \mathrm{MHz}$ in Fig. 1 show this most clearly. The highest compression in an SNR should occur just inside the forward shock, where the optical filaments form. Thus one expects the highest magnetic fields and highest synchrotron emissivity in the high compression regions. This now explains the observed spectral index variations: higher magnetic field at larger radius within the SNR produces flatter radio spectrum for the outer regions. Thus a larger proportion of steep spectrum emission for lines-of-sight through the central body of the SNR results in an observed steeper spectral index for interior regions. The conclusion is consistent with the explanation given by Leahy et al. (1998) for spectral index variations at HB9, although that study did not do a comparison of limb and interior spectral index values.

The study of Leahy et al. (1998) used the old DRAO $408 \mathrm{MHz}$ and $1420 \mathrm{MHz}$ observations and combined those with existing $2695 \mathrm{MHz}$ and $4850 \mathrm{MHz}$ data from the Effelsberg telescope, observations at $151 \mathrm{MHz}$ with the Cambridge LowFrequency synthesis telescope and $232 \mathrm{MHz}$ observations with the Beijing Astronomical Observatory Miyun telescope. The 151 and $232 \mathrm{MHz}$ maps suffered from lack of low-order spacings, and the mean derived spectral indices for $151-232 \mathrm{MHz}$ and 232-408 MHz indicate that the brightness scale for $232 \mathrm{MHz}$ map was too large. To compare the effect on spectral index of the improved 408 and $1420 \mathrm{MHz}$ data, we show the old 408-1420 MHz spectral index map in Fig. 6 (right panel) beside the spectral index map derived from the new data. The old map has an important artifact produced by the point source: the large dark area near RA 4 H55M, Dec $+46 \mathrm{~d} 20 \mathrm{~m}$ is due to $4 \mathrm{C} 46.09$. The contribution removed in that analysis was apparently too small to take into account the high brightness of 4C46.09. The same artifact affects the spectral index map made using the new data with CS included, but not in the spectral index map (shown in the left panel of Fig. 6) with CS subtracted. The old and new spectral index maps are globally similar (e.g. the limb has a flatter spectral index than the interior, with the lowest index coming from the bright southeast filament). There are, however, significant differences in the details between the two spectral index maps. This might be attributed to the sensitivity of the automatic fitting routine to small changes: for example compare case 1 (automatic fit) and case 3 (manual fit) in Table 1. It is interesting to note that the frequency-averaged spectral index map, which should suffer least from these errors, bears similarity to the results presented here: steeper index from the central regions than the rim.

\section{Conclusion}

We present new higher sensitivity and higher resolution images of the SNR HB9 at $408 \mathrm{MHz}$ and $1420 \mathrm{MHz}$, and study its radio spectrum, corrected for compact source flux densities using new improved methods. The T-T plot spectral index for HB9 agrees with the integrated flux-density based $408-1420 \mathrm{MHz}$ spectral index. A study of spatial variations shows no systematic difference in spectral index between weak and strong filaments, supporting the conclusion of Leahy \& Roger (1991). We find steeper spectral index for interior regions than for rim regions. This can be explained by a standard curved interstellar electron energy spectrum combined with variable magnetic field. Due to lower compression, a lower magnetic field in the interior, compared to that near the outer shock, results in steeper spectrum emission from the interior. Thus a larger proportion of steep spectrum emission for lines-of-sight through the central body of the SNR results in an observed steeper spectral index for interior regions. Based on $\mathrm{HI}$ features associated with $\mathrm{HB}$ 9, we obtain a distance of $0.8 \pm 0.4 \mathrm{kpc}$, and give updated Sedov and evaporating cloud model parameters for the SNR. We discuss the possible association between the pulsar and HB9, and conclude that more evidence is necessary to decide this possible association.

Acknowledgements. We acknowledge support from the Natural Sciences and Engineering Research Council of Canada. WWT thanks the NSFC for support. The DRAO is operated as a national facility by the National Research Council of Canada. The Canadian Galactic Plane Survey is a Canadian project with international partners.

\section{References}

Alvarez, H., Aparici, J., \& Reich, P. 2001, A\&A, 372, 636

Caswell, J. L., \& Lerche, I. 1979, MNRAS, 187, 201

Cox, D. 1972, ApJ, 178, 159

Fürst, E., Reich, W., Reich, P., \& Reif, K. 1990, A\&AS, 85, 691

Haslam, C. G. T., Salter, C. J., Stoffel, H., \& Wilson, W. W. 1982, A\&AS, 47, 1 Higgs, L. A., \& Tapping, K. F. 2000, AJ, 120, 2471

Leahy, D. A. 1987, ApJ, 322, 917

Leahy, D. A. 2006, ApJ, 647, 1125

Leahy, D. A., \& Aschenbach, B. 1995, A\&A, 293, 853

Leahy, D. A., \& Roger, R. S. 1991, ApJ, 101, 1033

Leahy, D. A., \& Roger, R. S. 1998, ApJ, 505, 784

Leahy, D. A., \& Tian, W. W. 2005, A\&A, 440, 929

Leahy, D. A., Zhang, X. Z., Wu, X. J., \& Lin, J. L. 1998, A\&A, 339, 601

Lozinskaya, T. A. 1981, SvAL, 7, 17

Manchester, R. N., Hobbs, G. B., Teoh, A., \& Hobbs, M. 2005, AJ, 129, 1993

Milne, D. K. 1979, AuJPh, 32, 83

Reich, W., Reich, P., \& Fürst, E. 1990, A\&AS, 83, 539

Reich, W., Reich, P., \& Fürst, E. 1997, A\&AS, 126, 413

Taylor, A. R., Gibson, S. J., Peracaula, M., et al. 2003, AJ, 125, 3145

Tian, W. W., \& Leahy, D. A. 2005, A\&A, 436, 187

Uyaniker, B., Reich, W., Yar, A., \& Fürst, E. 2004, A\&A, 426, 909

White, R., \& Long, K. 1991, ApJ, 373, 543

Zhang, X., Zheng, Y., Landecker, T., \& Higgs, L. 1997, A\&A, 324, 641 